\title{
RETROGRADATION PROPERTIES OF HEAT MOISTURE TREATED (HMT) SAGO AND ARENGA STARCHES
}

\author{
Dede R. Adawiyah ${ }^{1 *}$, Tomoko Sasaki ${ }^{2}$, Kaoru Kohyama ${ }^{2}$
}

\author{
${ }^{1}$ Department of Food Science and Technology, Faculty of Agricultural Technology, Bogor Agricultural University, \\ Bogor 16002, Indonesia. \\ ${ }^{2}$ National Agriculture and Food Research Organization, NARO, 2-1-12 Kannondai, Tsukuba, 305-8642, Japan. \\ ${ }^{*}$ Correspondence Email: dede_adawiyah@apps.ipb.ac.id
}

\begin{abstract}
This study aimed to investigate the retrogradation rate of heat moisture treated sago and arenga starches using different approaches, including a thermal approach using DSC (differential scanning calorimetry), a rheological approach using dynamic viscoelasticity as rheological and syneresis level. The autoclaving procedures prepared the HMT starches at $20 \%$ moisture content and warmed to $120^{\circ} \mathrm{C}$ for $60 \mathrm{~min}$ and $90 \mathrm{~min}$ for sago and arenga starches, respectively. The Avrami equation was used to express starch retrogradation kinetics based on gelatinization enthalpy $(\Delta \mathrm{H})$. The Avrami exponent $(\mathrm{n})$ of HMT and native starches were close to $1.0(0.77-1.20)$ indicates rapid nuclei growth of the crystal. HMT has a significant influence on the retrogradation of sago starch, both from the values of $\mathrm{n}$ and $\mathrm{k}$ of the Avrami equation. On the other hand, it does not have a significant effect on arenga starch. Based on the thermal approach (DSC), HMT significantly affects sago starch's retrogradation rate, but there was no effect on arenga starch. The influence of HMT on the retrogradation rate of arenga starch was observed on rheology and syneresis approaches, although it was not as high as sago starch.
\end{abstract}

Keywords: enthalpy, syneresis, avrami equation, rheology

\section{INTRODUCTION}

Retrogradation of starch is a term used for the alteration that happens during starch gelatinization, in which the starch transforms from an amorphous state, proceeding to a more organized or crystalline state. The changes during retrogradation are manifested by rheological properties (increasing firmness or rigidity), crystallinity, and waterholding capacity (syneresis). Retrogradation's effects on a starch-based product can be desirable, though it is sometimes unwanted. It has undesirable effects since retrogradation in starch plays a role in staling of bread and other starch-rich ingredients, leading to shortened shelflife and decreasing consumer acceptance. Retrogradation could alter the structural, mechanical, or organoleptic attributes of several starch-based products. However, starch retrogradation is desirable in some applications, including the production of breakfast cereal, parboiled rice, dehydrated mashed potatoes, and rice vermicelli, and may also be desirable in terms of nutrition due to slower digestion, the moderated release of glucose into the bloodstream, and/or low glycemic indices (Cahyana et al., 2019; Wang et al., 2019).

Heat moisture treatment (HMT) is described as a physical alteration that includes low moisture levels and heating at high temperatures. Various researchers have reported that HMT modified the physical characteristics of starch. Zavareze and Dias (2011) reviewed HMT's impact and annealing in starches on swelling power and solubility, pasting properties, gel texture, granule morphology, crystallinity, gelatinization characteristics, and susceptibilities to enzymes and acids. Adawiyah et al. (2017) reported HMT processing of sago and arenga starches using the autoclaving procedures at $120^{\circ} \mathrm{C}$ and $20 \%$ moisture content for $60 \mathrm{~min}$ and $90 \mathrm{~min}$ for sago and arenga, respectively, reduced the gelatinization enthalpy
$\Delta \mathrm{H}$ and significantly changed the flow behavior (viscosity), viscoelasticity, and textural characteristics of starch gels.

HMT's effects on starch retrogradation rates have been investigated by some researchers with varied results. Takaya et al. (2000) reported that retrogradation ratios grew faster for heat moisture-treated corn starch than for one without HMT. Adebowale et al. (2005) showed that heat moisture treatment of starch increased retrogradation tendencies observed from an increase in the pasting profiles' setback values as measured with a Rapid Visco Analyzer (RVA). Meanwhile, Li et al. (2017) reported that HMT reduced the sweet potato starch retrogradation rate evaluated by the avrami equation.

This study aimed to examine the impact of heat moisture treatment of arenga and sago starches on retrogradation properties using several methods, including differential scanning calorimetric as a thermal approach, and dynamic viscoelasticity as rheological and synerestical determinations. In this study, sensitivities to heat and moisture treatment on retrogradation rates were compared between sago and arenga starches. The information obtained from this research can be used to select sago or arenga starches from the characteristic of starch retrogradation rate. Currently, starch retrogradation is desirable to improve nutritional properties to produce rapidly digestible starch (RDS), slowly digestible starch (SDS), or resistant starch (RS). Sago and arenga starches are different palm starch types with similar composition and amylose content but differ significantly in gelatinization, swelling power, and rheological properties (Adawiyah et al., 2013). The HMT influence on rheology and textural attributes was stronger in sago starch than arenga starch. 


\section{MATERIALS AND METHODS}

\section{Materials}

Arenga Samples of arenga (Arenga pinnata) starch was purchased from arenga starch industry in Sukabumi area, while Sago (Metroxyllon sago) starch was collected from Bogor area, West Java, Indonesia. The samples were then further refined through the process of washing, filtering, decantation, and drying. Heat moisture treatment was performed by autoclaving using optimum HMT conditions at a moisture level of $20 \%$, heating at $120^{\circ} \mathrm{C}$ for $60 \mathrm{~min}$ for sago starch and $90 \mathrm{~min}$ for arenga starch, and then drying overnight as mentioned in Adawiyah et al. (2017)

\section{Differential Scanning Calorimetry}

The thermal properties of retrograded starches were studied by using a differential scanning calorimeter DSC 120 (Seiko instrument, Japan). The silver pan containing 30\% solid (wet basis) was heated twice at $1{ }^{\circ} \mathrm{C} / \mathrm{min}$ and distilled water was used as a reference. The first heating run was conducted from $30^{\circ} \mathrm{C}$ to $130^{\circ} \mathrm{C}$. After first run, then pan was immediately cooled down and stored at $4^{\circ} \mathrm{C}$ at certain periods. The stored pan temperature was raised from 3 to $100^{\circ} \mathrm{C}$ at the same increasing rate $\left(1^{\circ} \mathrm{C} / \mathrm{min}\right)$. Each sample analysis was measured in triplicate. The degree of retrogradation (retrogradation ratio) was calculated as the ratio of the re-gelatinization enthalpy $\Delta H$ of retrograded starch after storage to gelatinization enthalpy of starch before storage.

The retrogradation ratio was then further analyzed by Avrami equation to describe the kinetics of starch recrystallization. The equation can be expressed as:

$$
\frac{\Delta H_{L}-\Delta H_{t}}{\Delta H_{L}-\Delta H_{0}}=\exp \left(-k t^{n}\right)
$$

In which $\Delta H_{\mathrm{L}}$ is the limiting enthalpy or saturated value of retrogradation enthalpy obtained after storage more than 14 days at $4^{\circ} \mathrm{C}, \Delta H_{t}$ is the value of retrogradation enthalpy at time $\mathrm{t}, \Delta H_{0}$ is the initial value at $t=0, n$ is Avrami exponent, $k$ is the crystal rate constant. Calculation of avrami exponents were performed triplicate in accordance with DSC primary data measurements.

\section{Rheology}

To study retrogradation by a rheological approach, starch gels $(10 \% \mathrm{w} / \mathrm{v})$ that were stored for 07 days at $4^{\circ} \mathrm{C}$ were subject to dynamic viscoelasticity measurements using a Rheometer (AR-G2, TA Instruments, New Castle, USA). Before the measurements, starch gels were equilibrated to room temperature $\left(23^{\circ} \mathrm{C}\right)$ for $1 \mathrm{~h}$. Elastic modulus $\left(G^{\prime}\right)$ was determined under a linear region (10-20 Pa oscillation stress) at a constant frequency of $1 \mathrm{~Hz}$ and a temperature of $25^{\circ} \mathrm{C}$. Each sample was measured five times.

\section{Syneresis}

Gel syneresis was determined according to Charoenrein et al. (2008) with some procedure modifications. Starch gel $10 \% \mathrm{w} / \mathrm{v}$ was prepared in centrifuge tubes and stored in refrigerator $4-5^{\circ} \mathrm{C}$ for $0-7$ days. The stored gels were then equilibrated at room temperature for $1 \mathrm{~h}$ before centrifugation at $3500 \mathrm{rpm}$ for $15 \mathrm{~min}$. Then the liquid was separated from the gel and the percentage of syneresis $(\%$ $\mathrm{w} / \mathrm{w})$ was deduced as the ratio of liquid separated from gel after centrifugation to gel weight before centrifugation. Syneresis measurement was conducted in duplicate.

\section{Statistical Analysis}

Statistical analysis was carried out using $t$-test (two tailed) for two paired samples (native and HMT starches) using Microsoft Excel at a significance level of 5\% $(p=0.05)$.

\section{RESULT AND DISCUSSION}

\section{Differential Scanning Calorimetry}

Figure 1 presents DSC curves of starch gelatinization from the first run (before storage) and re-gelatinization after storage within $1-7$ days at $4^{\circ} \mathrm{C}$. The endothermic curve of starch re-gelatinization after storage shifted to lower temperatures than was shown on the endothermic curve of starch before storage. Within 1-2 days of storage, DSC curves exhibited small changes in the baseline. After three days of storage, DSC endothermic curves start to form a wide curve or broad temperature range $(T \mathrm{o}-T \mathrm{c})$. Endothermic temperatures of retrograded starch occurred at lower temperature than those for gelatinization starch. Once the starch granules are disorganized by gelatinization, the organized structure cannot be retrieved again entirely even after storage at low temperature for an extended period. Retrogradation results in crystalline forms of starch that are distinct from those available in the native starch granules. During storage at low temperature, gelatinized starch molecules reassociate but in a less ordered manner, form less organized or stable forms than those existing in the native granule (Li et al., 2016).

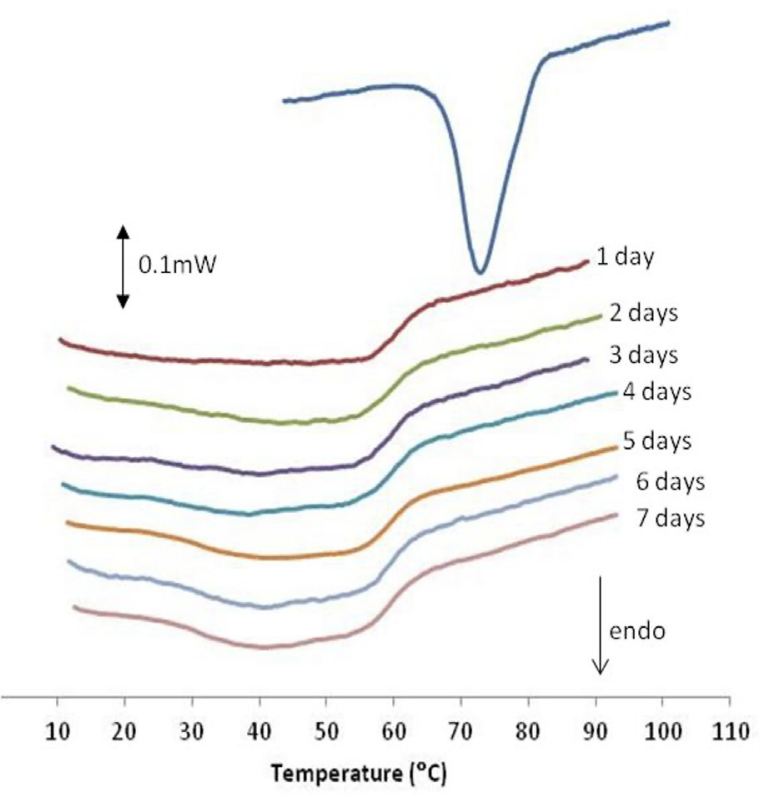

Figure 1. DSC curves of HMT sago starch from first run (before storage) and after storage for $1-7$ days at $4^{\circ} \mathrm{C}$. Moisture content: $70 \% \mathrm{db}$. Heating rate: $1^{\circ} \mathrm{C} / \mathrm{min}$ 
Table 1. Avrami exponent (n) and rate constant of HMT and native arenga and sago starches during storage at $4^{\circ} \mathrm{C}$

\begin{tabular}{lccccc}
\hline Starch & condition & $\mathbf{n}$ & $\mathbf{p}$ value & $\mathbf{k ( 1 / \text { day } )}$ & p value \\
\multirow{2}{*}{ Sago } & native & $1.20 \pm 0.078$ & $0.003 *$ & $0.28 \pm 0.017$ & $0.003^{*}$ \\
& HMT & $0.77 \pm 0.080$ & & $0.58 \pm 0.042$ & \\
\hline \multirow{2}{*}{ Arenga } & native & $0.97 \pm 0.149$ & \multirow{2}{*}{0.608} & $0.49 \pm 0.107$ & \multirow{2}{*}{0.73} \\
& HMT & $1.03 \pm 0.140$ & & $0.46 \pm 0.039$ & \\
\hline
\end{tabular}

Note: * significant different at $\alpha$ risk $5 \%$

The change in the retrogradation ratio showed an exponential linear relationship within five days after storage. The kinetics of starch retrogradation can be expressed with the Avrami equation. The Avrami equation has widely been employed to study the kinetics of starch retrogradation kinetics. The Avrami exponent (n) is associated with the mode of crystal nucleation. The Avrami exponent (n) of native sago and arenga starches were 1.27 and 0.97 , respectively, while for HMT sago and arenga starches were 0.77 and 1.03 (Table 1). The $n$ value of HMT sago starch was significantly higher than native, while there was no significant different between native and HMT arenga starches. The heat moisture treatment had a significant effect on retrogradation behavior of sago starch, while it had no effect on arenga starch. Li et al. (2017) reported the Avrami exponents of native and HMT-35 sweet potato starches were 0.70 and 0.98 , respectively. Li et al. (2016) reported that the values of the Avrami exponent (n) of all corn starches treated with 1,4-a-glucan branching enzyme were below 1(0.60-0.72), suggesting that the crystal nucleus occurred at once during recrystallization process. The $n$ value $\sim 1$ indicates that retrogradation has an instantaneous nuclei, followed by rod-like growth of crystals (McIver et al., 1968; Jouppila et al., 1998; Li et al., 2016). The values of the Avrami exponent $<1$ suggests that the crystal nucleus occurred immediately during the recrystallization process.

The Avrami constant $(\mathrm{k})$ can be defined as a retrogradation rate that reflects crystal growth and speed of crystallization. The retrogradation rate or $k$ value of HMT starch was higher than the native one, especially in sago starch. The HMT sago starch had a $\mathrm{k}$ value of $0.58 \pm 0.042$ /day that was significantly higher $(\mathrm{p}<0.05)$ than that of native sago $(\mathrm{k}=0.28 \pm 0.017 /$ day) (Table 1$)$. Meanwhile, $\mathrm{k}$ values after HMT in arenga starch was not significantly different from native $(\mathrm{p}>0.05)$. The retrogradation rate $(\mathrm{k})$ of HMT arenga starch was $0.46 \pm 0.039$, while native arenga starch was $0.49 \pm 0.107$ /day. The effect of HMT on the retrogradation rate was significant in sago starch, but there was no effect on arenga starch.

\section{Rheology}

Another indicator used to measure retrogradation is obtained by using the rheological approach. It is already known that retrogradation will promote starch gel firmness or rigidity. During storage, amylopectin recrystallizes, thus gel adding the rigidity, which in turn, reinforces the continuous amylose phase (Karim et al., 2000). HMT significantly raised the viscoelastic moduli $G^{\prime}$ and $G^{\prime}$. Figure 2 shows the increase in the storage modulus $G^{\prime}(\mathrm{Pa})$ of HMT and native arenga and sago starch gels at $10 \%$ (w/v) during storage at $4^{\circ} \mathrm{C}$. HMT promoted retrogradation for both arenga and sago starches. Retrogradation rates are estimated from the increase in $G^{\prime}(\mathrm{Pa})$ at the different days of storage (slope of linear trend). Retrogradation rate of native sago and arenga starches were 864 and $761 \mathrm{~Pa} /$ day, respectively. HMT starches showed higher retrogradation rate of $2897 \mathrm{~Pa}$ /day for HMT sago starch, and $1690 \mathrm{~Pa}$ /day for HMT arenga starch (Figure 2). HMT caused an increase of retrogradation rate of both starches. The increase in sago starch was higher (3.3 times) than arenga starch (2.2 times).

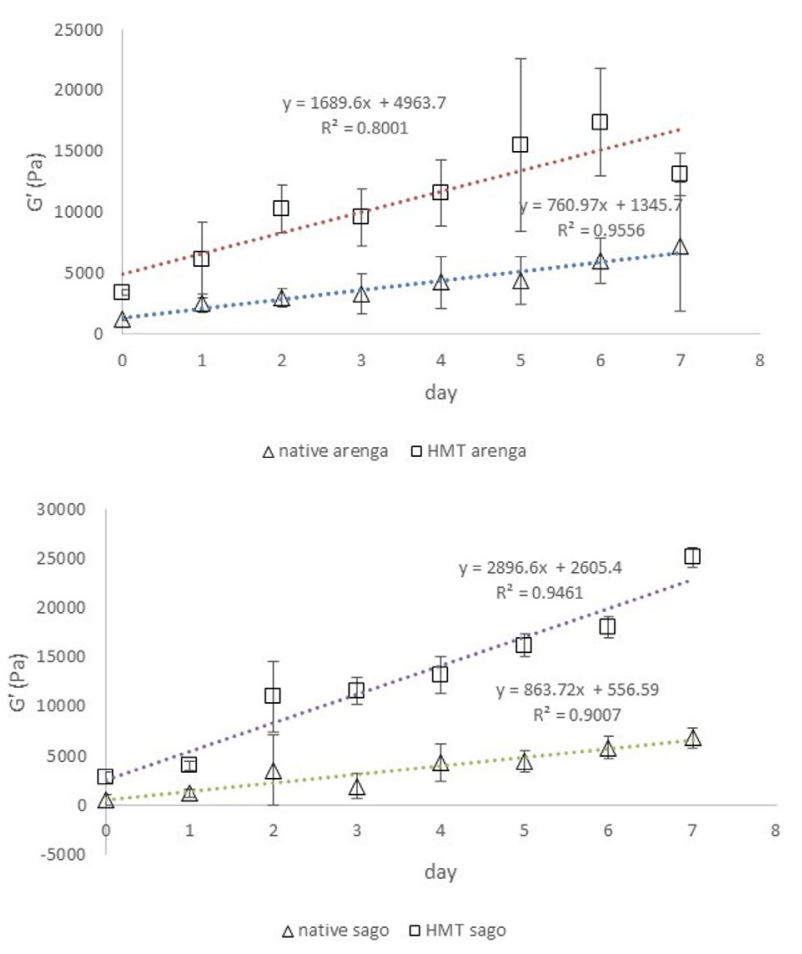

Figure 2. Elastic modulus, G', of $10 \%(\mathrm{w} / \mathrm{v})$ native and HMT arenga and sago starch gels during storage at $4^{\circ} \mathrm{C}$.

The results obtained in this rheology section differ from the results of thermal analysis (DSC) as mentioned above. The analysis using DSC, it was found that there was no significant difference in the retrogradation rate of native and HMT arenga starches. This difference is caused by different measurement mechanisms. Measurement of retrogradation properties using DSC is a microscopic level by measuring the development of the crystallinity of starch through $\Delta \mathrm{H}$ values. The $\Delta \mathrm{H}$ value provide a quantitative measure of the energy transformation that occurs during the melting of recrystallized amylose during retrogradation. A rheology is a macroscopic approach by direct detection of mechanical behavior of starch gel network (Karim et al., 2000). Adawiyah et al. (2017) reported that the HMT 
process did not significantly influence the amylopectin chain-length distribution in Arenga starch. HMT in the sago starch affects long-chain amylopectin with degree of polymerization of $\mathrm{C} \geq 37$. Therefore, structural rearrangement of starch caused by HMT, especially in sago starch, facilitates an increase in retrogradation rate. The combination of thermal and rheological methods is highly recommended in studying starch retrogradation.

HMT starch gels showed syneresis that was observed visually, suggesting loss of water holding capacity. Syneresis levels in HMT sago starch gel were more intense than those in HMT arenga and were visually detectable on the first day of storage.

\section{Syneresis}

Syneresis was determined by measuring the amount of water expelled during storage at low temperatures. Recrystallization of amylose after gelatinization caused phase separation, and water molecules are released from the dense matrix. Figure 3 shows the syneresis rate of sago and arenga starches (native and HMT) for seven days storage at $4-5^{\circ} \mathrm{C}$.

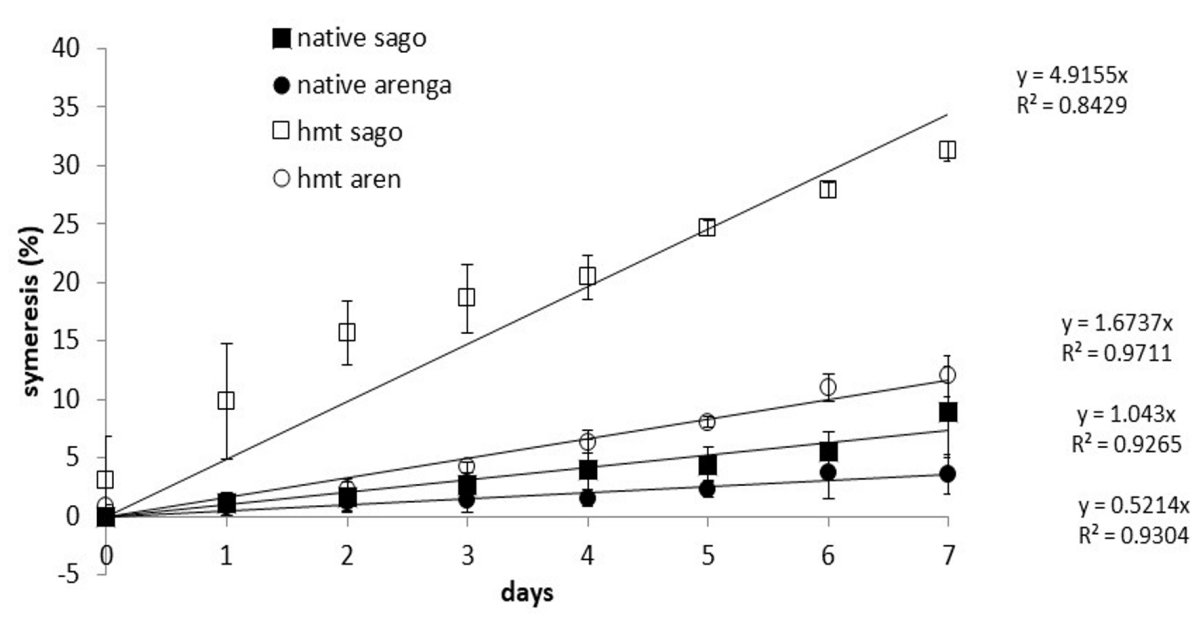

Figure 3. Level of syneresis Native and HMT sago and arenga starches

In native starch, the syneresis rate of native sago starch was higher (1.04\%/day) than that of arenga starch $(0.52 \% /$ day). HMT starches shows significantly higher syneresis rate than native starches. The syneresis rate of HMT sago starch was $4.92 \%$ /day (4.7 times of native), and HMT arenga starch was $1.67 \%$ /day (3.2 times of native). The starch modification using HMT altered starch properties recrystallization occurred during HMT process. Adawiyah et al. (2017) reported that HMT had a very significant impact on textural or mechanical attributes of arenga and sago starch gels. The HMT of sago and arenga starch gels were more rigid but more fragile (prone to break or fracture) than that of the native starches. These changes were probably caused by changes in physical and structural arrangements of amylose regions during heating under conditions of restricted moisture content.

A similar pattern using rheological approaches was observed with respect to syneresis levels. Sago showed a higher syneresis rate in comparison to arenga starch. Sago starch was more sensitive to heat and moisture treatment and had more significant effects in the retrogradation rate than that of arenga starch. Sago and arenga starches have comparable amylose content and peak gelatinization temperature (Tp), but these two starches have different rheological properties. HMT's effects on rheological attributes and texture of the sago starch were more significant than the changes recognized in arenga starch, as mentioned above. The HMT process did not significantly affect the amylopectin chainlength distribution in arenga starch, but in the sago starch,
HMT caused slight but significant changes to long-chain amylopectin with DP 337.

Overall, the HMT sago starch showed the highest retrogradation rate by all approaches used (thermal, rheology, and syneresis). The sago starch shows good potential in producing retrograded starch for specific purposes such as RS (resistant starch). Cahyana et al. (2019) proved that HMT markedly modified the pasting properties of bananas starch and resulted in the highest SDS (slowly digestible starch).

\section{CONCLUSION}

Based on the thermal approach (DSC), HMT affects significantly the retrogradation rate of sago starch, but there was no effect on arenga starch. The influence of HMT on the retrogradation rate of arenga starch was observed on rheology and syneresis approaches, although it was not as high as sago starch. The combination of microscopic technique and structural approach using DSC and macroscopic techniques such as rheological and syneresis methods are highly recommended in studying starch retrogradation.

\section{ACIKNOWLEDGMENT}

The first author gratefully acknowledges the Kirin Holding Co. Japan and United Nation University (UNU) for awarding research fellowship program at NFRI-NAROJapan. 


\section{REFERENCES}

Adawiyah, D. R., Sasaki, T., and Kohyama, K. 2013.Characterization of arenga starch in comparison with sago starch. Carbohydrate Polimers 92: 2306-2313.

Adawiyah, D. R., Akuzawa, S., Sasaki, T., and Kohyama, K. 2017. A comparison of the effect of heat moisture treatment (HMT) on rheological properties and amylopectin structure in sago (Metroxylon sago) and arenga (Arenga pinnata) starches. Journal of Food Science and Technology. 54(11):3404-3410.

Adebowale, K. O., Henle, T., Schwarzenbolz, U., and Doert, T. 2009. Modification and properties of African yam bean (Sphenostylis stenocarpa Hochst. Ex A. Rich) Harms starch I: heat moisture treatment and annealing. Food Hydrocolloids 23: 1947-1957.

Cahyana Y., Wijaya E., Halimaha T.S., Martab H., Suryadic E., Kurniatia D. 2019. The effect of different thermal modifications on slowly digestible starch and physicochemical properties of green banana flour (Musa acuminata colla). Food Chemistry 274: 274-280.

Charoenrein S., Tatirat O., and Muadklay J. 2008. Use of CentrifugationFiltration for Determination of Syneresis in Freeze-thaw Starch Gels. Carbohydrate Polymer 73: 143-147

Jouppila, K., Kansikas, J., Ross, Y.H. 1998. Factors affecting crystallization and crystallization kinetics in amorphous corn starch. Carbohydrate Polymers, 36: 143-149
Karim, A. A., Norziah, M. H., and Seow, C. C. 2000. Review: methods for the study of starch retrogradation. Food Chemistry 71: 9-36.

Li, W., Li, C., Gu, Z., Qiu, Y., Cheng, L., Hong, Y., Li, Z. 2016. Retrogradation behavior of corn starch treated with 1,4-a-glucan branching enzyme. Food Chemistry 203:308-313.

Li, Y., Liu, S., Liu, X., Tang, X., Zhang, J. 2017. The impact of heatmoisture treatment on physicochemical properties and retrogradation behavior of sweet potato starch. International Journal of Food Engineering. 20170001

McIver, R. G., Axford, D. W. E., Colwell, K. H., and Elton, G. A. 1968. Kinetic study of the retrogradation of gelatinized starch. Journal of Science, Food and Agriculture 19: 560-563.

Takaya, T., Sano, C., and Nishinari, K. 2000. Thermal studies on the gelatinization and retrogradation of heat-moisture treated starch. Carbohydrate Polymers 41: 97-100.

Wang M., Muqi Sun M., Zhang Y., Chen Y., Wu Y., Ouyang J. 2019. Effect of microwave irradiation-retrogradation treatment on the digestive and physicochemical properties of starches with different crystallinity. Food Chemistry 298: 125015

Zavareze, E. R., and Dias, A. R. G. 2011. Impact of heat moisture treatment and annealing in starches: A review. Carbohydrate polymers 83: 317 328. 